\title{
POINTWISE APPROXIMATION BY BERNSTEIN POLYNOMIALS
}

\author{
GANCHO TACHEV
}

\author{
(Received 22 April 2011)
}

\begin{abstract}
We improve the degree of pointwise approximation of continuous functions $f(x)$ by Bernstein operators, when $x$ is close to the endpoints of $[0,1]$. We apply the new estimate to establish upper and lower pointwise estimates for the test function $g(x)=x \log (x)+(1-x) \log (1-x)$. At the end we prove a general statement for pointwise approximation by Bernstein operators.
\end{abstract}

2010 Mathematics subject classification: primary 41A10; secondary 41A15, 41A25, 41A36.

Keywords and phrases: Bernstein polynomials, Direct theorems, Ditzian-Totik moduli of smoothness.

\section{Introduction}

In 1994 Ditzian showed in [4] that for the Bernstein polynomials

$$
B_{n}(f ; x)=\sum_{k=0}^{n} f\left(\frac{k}{n}\right) \cdot\left(\begin{array}{l}
n \\
k
\end{array}\right) x^{k}(1-x)^{n-k}, \quad x \in[0,1],
$$

the pointwise approximation

$$
\left|B_{n}(f, x)-f(x)\right| \leq C \omega_{2}^{\varphi^{\lambda}}\left(f, n^{-1 / 2} \varphi(x)^{1-\lambda}\right), \quad x \in[0,1],
$$

holds true for $\lambda \in[0,1], \varphi(x):=\sqrt{x(1-x)}$ and $f \in C[0,1]$, where the Ditzian-Totik modulus of second order is given by

$$
\omega_{2}^{\varphi^{\lambda}}(f, t):=\sup _{0<h \leq t} \sup _{x \pm h \varphi^{\lambda}(x) \in[0,1]}\left|f\left(x-h \varphi^{\lambda}(x)\right)-2 f(x)+f\left(x+h \varphi^{\lambda}(x)\right)\right| .
$$

We recall that this modulus is equivalent to the $K$-functional

$$
K_{\varphi^{\lambda}}\left(f, t^{2}\right)=\inf \left(\|f-h\|_{C[0,1]}+t^{2}\left\|\varphi^{2 \lambda} h^{\prime \prime}\right\|_{C[0,1]}\right) .
$$

The infimum is taken on functions satisfying $h \in A C, h^{\prime} \in A C_{\text {loc }}$ where $A C$ is the set of all absolutely continuous functions on $[0,1]$ and $A C_{\text {loc }}$ is the set of absolutely continuous functions on compact subsets of $(0,1)$. (See [5].)

(C) 2012 Australian Mathematical Publishing Association Inc. 0004-9727/2012 \$16.00 
In 1998 Felten proved in [6] the more general inequality

$$
\left|B_{n}(f, x)-f(x)\right| \leq C \omega_{2}^{\phi}\left(f, n^{-1 / 2} \frac{\varphi(x)}{\phi(x)}\right), \quad x \in[0,1],
$$

where $\phi:[0,1] \rightarrow \mathbb{R}$ is an admissible step-weight function of the Ditzian-Totik modulus and $\phi^{2}$ is a concave function. The aim of this note is to improve the estimate (1.1) for $\lambda=1$, when $x$ is close to the endpoints of $[0,1]$.

Let us define

$$
\delta(n, x):=\min \left\{n^{-1 / 2},\left(\frac{x(1-x)}{n}\right)^{1 / 4}\right\} .
$$

The following theorem is our main result.

THEOREM 1.1. The pointwise estimate

$$
\left|B_{n}(f, x)-f(x)\right| \leq C \omega_{2}^{\varphi}(f, \delta(n, x)), \quad x \in[0,1],
$$

holds true for all $f \in C[0,1], n \in \mathbb{N}$.

In Section 2 we give the proof of Theorem 1.1. In Section 3 we establish upper and lower bounds for approximation of the function $g(x)$, defined in (2.1), by Bernstein operators.

\section{Proof of Theorem 1.1}

Let us define $g:[0,1] \rightarrow \mathbb{R}$ as

$$
g(x)=x \log (x)+(1-x) \log (1-x), \quad x \in(0,1),
$$

and $g(0)=g(1):=0$. The problem of evaluating the remainder term

$$
R_{n}(g, x)=B_{n}(g, x)-g(x), \quad x \in[0,1],
$$

was formulated by the author in [14] during the fifth Romanian-German Seminar on Approximation Theory, held in Sibiu, Romania, in 2002. More precisely, we proposed to find (best) bounds of the type

$$
k_{1} \cdot \frac{x^{\alpha_{1}}(1-x)^{\alpha_{2}}}{n^{\beta}} \leq R_{n}(g, x) \leq K_{2} \cdot \frac{x^{a_{1}}(1-x)^{a_{2}}}{n^{b}}, \quad x \in[0,1],
$$

where $k_{1}, K_{2}$ are positive numbers, independent of $x$ and $n$. Some days after the conference, Lupaş showed that the above holds with $\alpha_{1}=\alpha_{2}=\beta=1, k_{1}=\frac{1}{2}$ and $a_{1}=a_{2}=b=\frac{1}{2}, K_{2}=\sqrt{2}$ (see $\left.[8,9]\right)$, that is,

$$
\frac{x(1-x)}{2 n} \leq R_{n}(g, x) \leq \sqrt{2} \cdot \sqrt{\frac{x(1-x)}{n}} .
$$

The function $g$ was applied in the following direct estimate, proved by Parvanov and Popov in [12]. 
If $L: C[0,1] \rightarrow C[0,1]$ is a linear positive operator, preserving linear functions, then

$$
|L(f, x)-f(x)| \leq 2\|f-h\|_{C[0,1]}+|L(g, x)-g(x)| \cdot\left\|\varphi^{2} h^{\prime \prime}\right\|_{C[0,1]}
$$

holds for arbitrary $h \in A C, h^{\prime} \in A C_{\text {loc }},\left\|\varphi^{2} h^{\prime \prime}\right\|_{C[0,1]}<\infty$. Instead of $L$ we write $B_{n}$ and apply the right-hand side of (2.2). Hence

$$
\left|B_{n}(f, x)-f(x)\right| \leq 2\|f-h\|_{C[0,1]}+\sqrt{2}\left(\frac{x(1-x)}{n}\right)^{1 / 2} \cdot\left\|\varphi^{2} h^{\prime \prime}\right\|_{C[0,1]} .
$$

Therefore

$$
\left|B_{n}(f, x)-f(x)\right| \leq 2 K_{\varphi}\left(f,\left(\frac{x(1-x)}{n}\right)^{1 / 2}\right) .
$$

From the equivalence between $K_{\varphi}\left(f, t^{2}\right)$ and $\omega_{2}^{\varphi}(f, t)$, it follows that

$$
\left|B_{n}(f, x)-f(x)\right| \leq C \omega_{2}^{\varphi}\left(f,\left(\frac{x(1-x)}{n}\right)^{1 / 4}\right) .
$$

The estimates (2.3) and (1.1) with $\lambda=1$ complete the proof.

\section{Upper and lower pointwise bounds}

The following is a straightforward corollary of Theorem 1.1.

Corollary 3.1. The pointwise estimate

$$
\left|B_{n}(g, x)-g(x)\right| \leq C \omega_{2}^{\varphi}\left(g, \sqrt[4]{\frac{x(1-x)}{n}}\right), \quad x \in[0,1],
$$

holds true for all $n \in \mathbb{N}$.

REMARK 3.2. If $x$ is close to the endpoints of $[0,1]$, then the estimate (3.1) is better than that in (2.1) for $\lambda=1$, established by Ditzian in [4].

REMARK 3.3. Other direct pointwise estimates in terms of $K_{\varphi}$ are proved in [6]. We point out that neither from [6] nor from [4] is it possible to deliver (3.1) as a straightforward corollary.

We continue with lower pointwise bounds. In [1, Theorem 11], using the function $g(x)$ as a 'universal' tool, the authors proved that

$$
c(g) \omega_{2}\left(g, \sqrt{\frac{x(1-x)}{n}}\right) \leq\left|B_{n}(g, x)-g(x)\right|
$$

does not hold. So the question arises: what kind of modulus is appropriate to serve as a lower pointwise bound for $\left|B_{n}(g, x)-g(x)\right|$ ? The answer is given in the next theorem.

TheOREM 3.4. The following inequality holds true:

$$
c \cdot \omega_{2}^{\varphi}\left(g, \sqrt{\frac{x(1-x)}{n}}\right) \leq\left|B_{n}(g, x)-g(x)\right| .
$$


Proof. Using the equivalence between $K_{\varphi}\left(g, t^{2}\right)$ and $\omega_{2}^{\varphi}(g, t)$, we compute

$$
\begin{aligned}
c \omega_{2}^{\varphi}\left(g, \sqrt{\left.\frac{x(1-x)}{n}\right)}\right. & \leq K_{\varphi}\left(g, \frac{x(1-x)}{n}\right) \\
& :=\inf _{h}\left\{\|g-h\|_{C[0,1]}+\frac{x(1-x)}{n} \cdot\left\|\varphi^{2} h^{\prime \prime}\right\|_{C[0,1]}\right\} \\
& \leq\|g-g\|_{C[0,1]}+\frac{x(1-x)}{n} \cdot\left\|\varphi^{2} g^{\prime \prime}\right\|_{C[0,1]} \\
& =\frac{x(1-x)}{n} \leq 2\left|B_{n}(g, x)-g(x)\right|,
\end{aligned}
$$

where the last inequality follows from (2.2). The proof is complete.

REMARK 3.5. It was pointed out in [1] that for $f(x)=x^{3}, x \in[0,1]$, an estimate similar to (3.2) is not possible.

Remark 3.6. Theorems 3.4 and 3.7 imply for the function $g(x)$ in (2.1) the two-sided pointwise inequality

$$
c \omega_{2}^{\varphi}\left(g, \sqrt{\frac{x(1-x)}{n}}\right) \leq\left|B_{n}(g, x)-g(x)\right| \leq C \omega_{2}^{\varphi}\left(g, \sqrt[4]{\frac{x(1-x)}{n}}\right) .
$$

Very recently, motivated by the result of Lupas and considerations set out in [1, 2 , 12] we proved in [15] that the values of $\alpha_{1}=\alpha_{2}=1$ and $a_{1}=a_{2}=\frac{1}{2}$ in (1.4) are optimal, that is, we proved the following result.

THEOREM A. It is not possible to find $a_{1}>\frac{1}{2}$, or $a_{2}>\frac{1}{2}$, or $\alpha_{1}<1$, or $\alpha_{2}<1$, such that

$$
k_{1} \cdot \frac{x^{\alpha_{1}}(1-x)^{\alpha_{2}}}{n} \leq R_{n}(g, x) \leq K_{2} \cdot \frac{x^{a_{1}}(1-x)^{a_{2}}}{\sqrt{n}}
$$

holds true for all $x \in[0,1]$ with some positive numbers $k_{1}, K_{2}$, independent of $x$ and $n$.

Our next statement is the following theorem.

THEOREM 3.7. In both sides of (3.3) it is not possible to put one and the same modulus: neither $\omega_{2}^{\varphi}(g, \sqrt{x(1-x) / n})$ nor $\omega_{2}^{\varphi}(g, \sqrt[4]{x(1-x) / n})$.

Proof. First we suppose that $\omega_{2}^{\varphi}(g, \sqrt[4]{x(1-x) / n})$ could be placed in the left-hand side of (3.3). Setting $x=\frac{1}{2}$ in (1.2), we obtain

$$
\Delta_{h \varphi}^{2} g\left(\frac{1}{2}\right)=h^{2} \cdot \varphi^{2}\left(\frac{1}{2}\right) \cdot g^{\prime \prime}(\xi) \geq h^{2} \cdot\left(\frac{1}{2}\right)^{2} \cdot \frac{1}{\frac{1}{2}\left(1-\frac{1}{2}\right)}=h^{2} .
$$

Hence by

$$
t:=\sqrt[4]{\frac{x(1-x)}{n}}, \quad x \in[0,1] \text { fixed }
$$

we have

$$
\omega_{\varphi}^{2}(g, t) \geq t^{2}=\sqrt{\frac{x(1-x)}{n}} .
$$


From our supposition and the last inequality we get

$$
c \sqrt{\frac{x(1-x)}{n}} \leq\left|B_{n}(g, x)-g(x)\right|,
$$

which contradicts the statement of Theorem A (left-hand side of the inequality, as $x \rightarrow 0)$. Also if we suppose that $\omega_{2}^{\varphi}(g, \sqrt{x(1-x) / n})$ could be placed in the right-hand side of (3.3) due to the fact that (see [3, Theorem 6.1])

$$
\omega_{2}^{\varphi}(g, t) \leq C t^{2}\left\|\varphi^{2} g^{\prime \prime}\right\|=C t^{2} \cdot 1,
$$

the last inequality would imply that

$$
\left|B_{n}(g, x)-g(x)\right| \leq C \frac{x(1-x)}{n},
$$

which again contradicts Theorem A (right-hand side of the inequality, as $x \rightarrow 0$ ). The proof of Theorem 3.7 is complete.

REMARK 3.8. The upper pointwise bound in (3.1) in terms of the classical modulus of continuity $\omega_{2}(g, \sqrt{x(1-x) / n})$ was first established in [13]. As already mentioned, this modulus is not appropriate as a lower bound.

It is known that for the 'test' function $f_{1}(x)=x^{2}, x \in[0,1]$,

$$
B_{n}\left(f_{1}, x\right)-f_{1}(x)=\frac{x(1-x)}{n} \approx \omega_{2}\left(f_{1}, \sqrt{\frac{x(1-x)}{n}}\right) .
$$

What is the situation for all other continuous functions $f(x)$ ? In response to this question, we formulate the following result.

THeOREM 3.9. There are no constants $c(f)$ and $C(f)$ such that

$$
c(f) \Omega_{2}(f, \sigma(n, x)) \leq\left|B_{n}(f, x)-f(x)\right| \leq C(f) \Omega_{2}(f, \sigma(n, x))
$$

holds true for all $f \in C[0,1]$, all $x \in[0,1]$ and all $n \in \mathbb{N}$ with appropriate constructive characteristic $\Omega_{2}(f, \cdot)$, where $\Omega_{2}(f, \cdot)$ satisfies the properties of second-order modulus of smoothness (or related $K$-functional) and argument $\sigma(n, x)$.

Proof. The proof follows immediately from Theorem A and (2.2) for $g(x)$. We fix $n \in \mathbb{N}$ and take $x \rightarrow 0$. If we suppose that (3.4) holds true, this would imply simultaneously that

$$
\begin{gathered}
\Omega_{2}(g, \sigma(n, x)) \leq k_{1} \frac{x(1-x)}{n} \quad \text { as } x \rightarrow 0, \\
\Omega_{2}(g, \sigma(n, x)) \geq K_{2} \sqrt{\frac{x(1-x)}{n}} \quad \text { as } x \rightarrow 0,
\end{gathered}
$$

with some positive constants $k_{1}, K_{2}$ independent of $n, x$, which is not possible. Hence (3.4) fails for $g(x)$. 
Remark 3.10. The case of 'norm' estimates is quite different. We mention here the well-known equivalence result of Knoop and Zhou for Bernstein operators, namely

$$
c \omega_{2}^{\varphi}\left(f, \frac{1}{\sqrt{n}}\right) \leq\left\|B_{n} f-f\right\|_{C[0,1]} \leq C \omega_{2}^{\varphi}\left(f, \frac{1}{\sqrt{n}}\right),
$$

established in 1994 in [7]. Similar strong converse inequalities are valid for many other linear positive operators.

\section{Acknowledgements}

This note is dedicated to the memory of late Professor Alexandru Lupaş, one of the organisers of Romanian-German Seminars on Approximation Theory and distinguished Romanian mathematician, teacher and friend.

\section{References}

[1] J.-D. Cao, H. Gonska and D. Kacsó, 'On the impossibility of certain lower estimates for sequences of linear operators', Math. Balkanica 19 (2005), 39-58.

[2] J.-D. Cao, H. Gonska and D. Kacsó, 'On the second order classical and (weighted) DitzianTotik moduli of smoothness', in: Mathematical Analysis and Approximation Theory, Proc. 6th Romanian-German Sem. Approx. Theory and its Appl., Baisoara, 2004 (eds. I. Gavrea et al.) (Mediamira Science Publisher, Cluj-Napoca, 2005), pp. 35-42.

[3] R. A. DeVore and G. G. Lorentz, Constructive Approximation (Springer, Berlin, 1993).

[4] Z. Ditzian, 'Direct estimate for Bernstein polynomials', J. Approx. Theory 79 (1994), 165-166.

[5] Z. Ditzian and V. Totik, Moduli of Smoothness (Springer, New York, 1987).

[6] M. Felten, 'Direct and inverse estimates for Bernstein polynomials', Constr. Approx. 14 (1998), $459-468$.

[7] H. B. Knoop and X.-1. Zhou, 'The lower estimate for linear positive operators (II)', Res. Math. 25 (1994), 315-330.

[8] A. Lupaş, 'On a problem proposed by G. Tachev', in: Mathematical Analysis and Approximation Theory, Proc. 5th Romanian-German Seminar (eds. A. Lupaş et al.) (Burg, Sibiu, 2002), p. 326.

[9] A. Lupaş, L. Lupaş and V. Maier, 'The approximation of a class of functions', in: Mathematical Analysis and Approximation Theory, Proc. 5th Romanian-German Seminar (eds. A. Lupaş et al.) (Burg, Sibiu, 2002), pp. 155-168.

[10] V. Maier, 'The $L_{1}$-saturation class of the Kantorovich operator', J. Approx. Theory 22 (1978), 227-232.

[11] V. Maier, 'A new proof for the approximation of the Log-function by Kantorovich polynomials in the $L_{p}$-norm', Rev. Anal. Numér. Théor. Approx. 28(2) (1999), 173-177.

[12] P. E. Parvanov and B. D. Popov, 'The limit case of Bernstein's operators with Jacobi-weights', Math. Balkanica 8(2-3) (1994), 165-177.

[13] T. Popoviciu, 'Sur l'approximation des fonctions convexes d'ordre superieur', Math. Cluj 10 (1935), 49-54.

[14] G. Tachev, 'Three open problems', in: Mathematical Analysis and Approximation Theory, Proc. 5th Romanian-German Seminar (eds. A. Lupaş et al.) (Burg, Sibiu, 2002), p. 329.

[15] G. Tachev, 'Approximation of logarithmic function by Bernstein operator', manuscript, submitted, 2011.

GANCHO TACHEV, Department of Mathematics, University of Architecture, Civil Engineering and Geodesy, BG-1046, Sofia, Bulgaria

e-mail: gtt_fte@uacg.acad.bg 